\title{
Surface Analysis of Polymers using Helium Ion Microscopy Coupled with Secondary Ion Mass Spectrometry (HIM-SIMS)
}

\author{
Artem A. Trofimov ${ }^{1,2}$, Matthias Lorenz ${ }^{1,2}$, Anton Ievlev ${ }^{1,2}$, Stephen T. King ${ }^{1,2}$, Olga S. Ovchinnikova ${ }^{1,2}$ \\ and Alex Belianinov ${ }^{1,2 *}$ \\ 1. Center for Nanophase Materials Sciences, Oak Ridge National Laboratory, Oak Ridge, TN, USA. \\ 2. The Institute for Functional Imaging of Materials, Oak Ridge National Laboratory, Oak Ridge, TN, \\ USA. \\ * Corresponding author: belianinova@ornl.gov
}

Polyolefins such as polypropylene (PP), polyethylene (PE), and their copolymers are important industrial materials, used in numerous applications, including oils, tires, fibers, and films. In many of these, chemistry and structure of the materials and their blends can play a crucial role on material performance. Previous studies aimed at distinguishing PP and PE [1-3], and correlating chemistry to morphology have been difficult and required measurements on multiple instruments. In this work, we utilize a helium ion microscope (HIM) equipped with a magnetic sector secondary ion mass spectrometer (SIMS) [4] to differentiate between PE, PP, and their blends in situ; offering both chemical and physical information on these polymers.

The HIM is based on a gas field ionization source operating with helium or neon gas and offers a straightforward approach to investigating the structure of various highly insulating samples without the use of a conductive coating [5]. Moreover, the recent incorporation of SIMS analytical capability to the HIM, allows correlative imaging using secondary electrons (SE) with the co-registered chemical information obtained from the SIMS [6].

Here, we demonstrate the HIM-SIMS to quantitatively differentiate between PE and PP, and their copolymer blends. We illustrate the detection, choice, and analysis of specific polymer fragments that allow us to distinguish between PE and PP (see example in Figure 1). Furthermore, we explore the influence of sample charging, depth profiling, as well as the field of view effect during the analysis. Additionally, the capability to correlate chemical and structural information of the polymer from the identical regions will be shown and discussed (see example in Figure 2) [7].

\section{References:}

[1] AA Galuska AA, Surface and Interface Analysis 25 (1997), pp. 1-4.

[2] FR Beamson and D Briggs, Surface and Interface Analysis 17 (1991), pp. 842-846.

[3] L Lianos, C Quet, and TM Duc, Surface and Interface Analysis 21 (1994), pp. 14-22.

[4] G Hlawacek and A Gölzhäuser eds. "Helium Ion Microscopy." (Cham: Springer).

[5] NBorodinov et al., Nanoscale 10 (2018), pp. 18001-18009.

[6] D Dowsett and T Wirtz, Analytical Chemistry 89 (2017), pp. 8957-8965.

[7] This research was supported by ExxonMobil Corporation and conducted at the Center for Nanophase Materials Sciences (CNMS), which is a DOE Office of Science User Facility. The authors acknowledge Drs. D. Abmayr, W. Lamberti, R. Colby, and S. Yakovlev from ExxonMobil Chemical Corporation for providing polymer samples and useful discussion. 


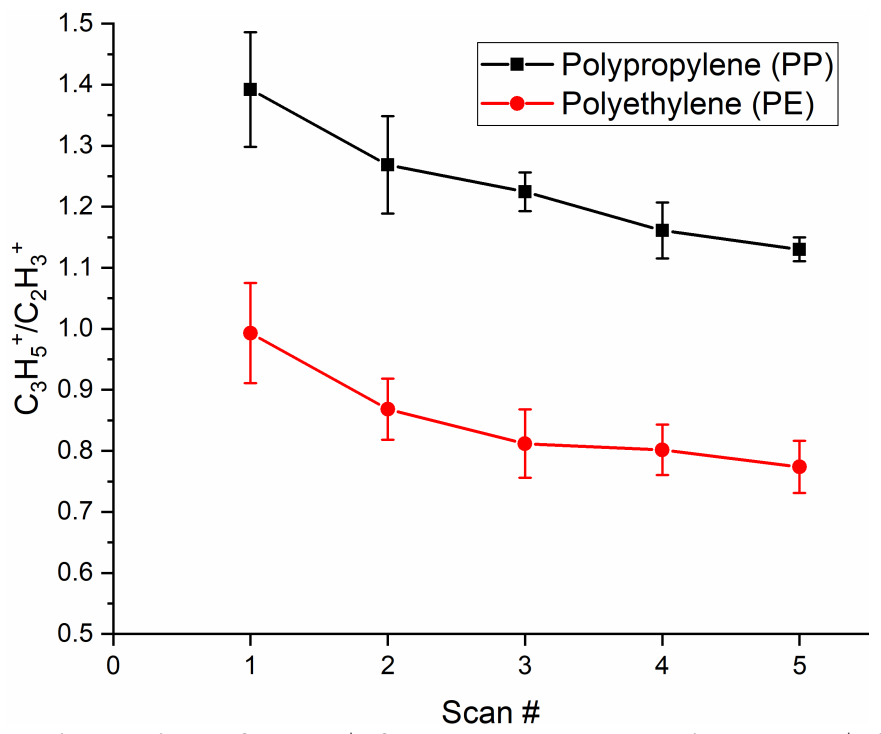

Figure 1. Plot of the relative intensity of $\mathrm{C}_{3} \mathrm{H}_{5}{ }^{+}$fragment compared to $\mathrm{C}_{2} \mathrm{H}_{3}{ }^{+}$fragment as a function of HIM-SIMS scans highlighting differentiation between PE and PP. Each point is an average of three measurements. Lines connecting the data points are for eye guidance only.
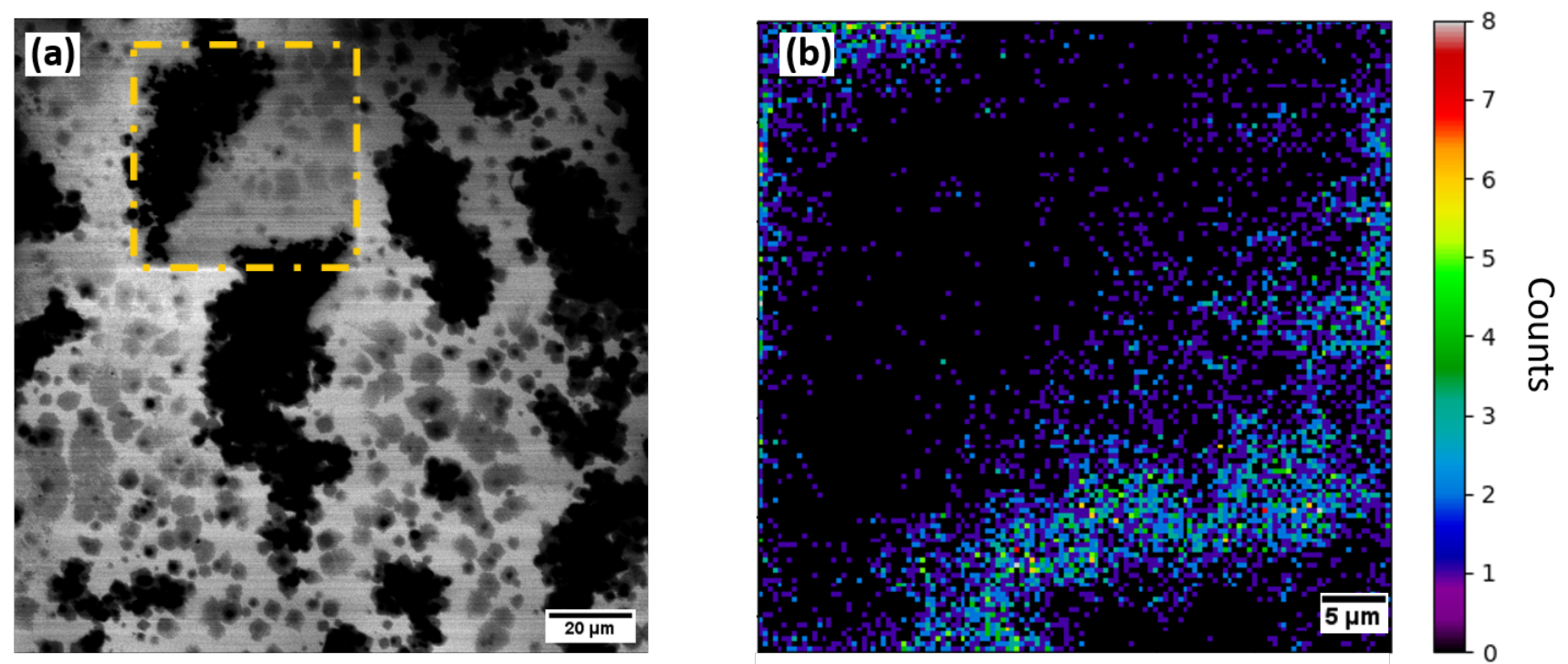

Figure 2. (a) SE image of PE obtained using HIM. (b) Chemical image of the $\mathrm{C}_{2} \mathrm{H}_{3}{ }^{+}$distribution in the area indicated in (a) obtained using HIM-SIMS. 